\title{
Comparison of the efficacy of colistin monotherapy and colistin combination therapies in the treatment of nosocomial pneumonia and ventilator-associated pneumonia caused by Acinetobacter baumannii
}

\author{
I Kara, ${ }^{1}$ MD; F Yildirim, ${ }^{2}$ MD; B Bilaloglu, ${ }^{3}$ MD; D Karamanlioglu, ${ }^{4}$ MD; E Kayacan, ${ }^{3} \mathrm{MD}$; M Dizbay, ${ }^{4} \mathrm{MD} ; \mathbf{M}$ Turkoglu, ${ }^{3} \mathrm{MD}$; \\ G Aygencel, ${ }^{3} \mathrm{MD}$ \\ ${ }^{1}$ Intensive Care Fellowship Programme, Department of Anesthesiology and Reanimation, Faculty of Medicine, Gazi University, Ankara, Turkey \\ ${ }^{2}$ Intensive Care Fellowship Programme, Department of Pulmonary Medicine, Faculty of Medicine, Gazi University, Ankara, Turkey \\ ${ }^{3}$ Division of Intensive Care Medicine, Department of Internal Medicine, Faculty of Medicine, Gazi University, Ankara, Turkey \\ ${ }^{4}$ Department of Infectious Diseases, Faculty of Medicine, Gazi University, Ankara, Turkey
}

Corresponding author: I Kara (driskenderkara@gmail.com)

Objective. To investigate whether there was a difference in mortality, clinical response and bacterial eradication between colistin monotherapy and colistin combination therapies for the treatment of nosocomial pneumonia/ventilator-associated pneumonia (VAP) caused by Acinetobacter baumannii in a medical intensive care unit (ICU).

Methods. This retrospective, observational and single-centre study included all patients who were in the medical ICU of Gazi University Medical Faculty Hospital and diagnosed with nosocomial pneumonia/VAP caused by A. baumannii between January 2009 and September 2014.

Results. The median age of the 134 patients was 68 years and $53.3 \%$ were male. The most common causes of admission were respiratory insufficiency (66.7\%) and sepsis/septic shock (54.8\%). In patients with nosocomial pneumonia/VAP caused by A. baumannii, on median day 5 of admission, colistin monotherapy was used in 23 (21.6\%) patients, a carbapenem combination was used in 80 (59.7\%) patients, sulbactam-ampicillin combination was used in 42 (31.4\%) patients, tigecycline combination was used in 26 (19.4\%) patients, and sulbactam-cefoperazone combination was used in 17 (12.7\%) patients. Median ICU stay of the patients was 15.5 days, and 112 (83.6\%) patients died. Colistin monotherapy and combination therapies had no superiority over each other in clinical response for the treatment of A. baumannii-associated nosocomial pneumonia/VAP. Mortality was found to be higher in patients receiving the colistin-carbapenem combination (64.3\% v. 36.4\%, $p=0.016$ ). Discharge/day-of-death Sequential Organ Failure Assessment score (odds ratio (OR) $2.017,95 \%$ confidence interval (CI) 1.330 - 3.061) and vasopressor use (OR 9.014,95\% Cl 1.360-59.464) were independent risk factors for ICU mortality. Conclusion. Colistin monotherapy and combination therapies have no superiority over each other for clinical response in the treatment of nosocomial pneumonia/VAP caused by multidrug-resistant $A$. baumannii. Colistin-SAM was associated with improved microbiological eradication and colistin-carbapenem combination was associated with increased mortality.

S Afr J Crit Care 2015;31(2):51-58. DOI:10.7196/SAJCC.2015.v31i2.246

Acinetobacter baumannii is an aerobic, Gramnegative, non-motile, lactose-negative, oxidasenegative, catalase-positive coccobacillus, and is a member of the Moraxellaceae family. ${ }^{[1,2]}$ Over the last 2 decades, nosocomial infections caused by multidrug-resistant (MDR) A. baumannii (MDR-AB) have increased globally. ${ }^{[3]}$ Infection by this agent increases mortality, morbidity and costs. Mortality rates can increase by up to $65 \%$ when pneumonias are caused by MDR-AB $\cdot{ }^{[4]}$ In critically ill patients who are in intensive care units (ICUs), MDR-AB infections are often difficult to treat since they are susceptible to only a limited number of antibiotics. Colistin, a polymyxin antibiotic, is one of the most common antibiotics used to treat MDR-AB infections. It is an important treatment option not just for MDR-AB infections, but also for other MDR Gram-negative bacterial infections. ${ }^{[5]}$ Colistin was first introduced in 1952 and was used routinely until the 1980 s. Its use was then abandoned for a period owing to serious side-effects such as nephrotoxicity and neurotoxicity; however, it then became popular again with the emergence of life-threatening MDR Gram-negative infections. Colistin, which is almost the only available treatment option for MDR Gram-negative infections, is often used in combination therapies in order to reduce resistance and improve efficacy. ${ }^{[6,7]}$

The primary objective of the study was to determine whether there was a mortality difference between colistin and colistin combination therapies in MDR-AB nosocomial pneumonia/ ventilator-associated pneumonia (VAP). Secondary objectives were to investigate clinical response, bacterial eradication and nephrotoxicity in colistin monotherapy and colistin combinations in MDR-AB associated pneumonia/VAP.

\section{Methods}

This retrospective, observational, single-centre study was conducted in the medical ICU of Gazi University Medical Faculty Hospital. Patients who were diagnosed with MDR-AB-associated nosocomial pneumonia/VAP between January 2009 and September 2014 were 
included. Patients' demographic characteristics, underlying diseases, other infection sites, dosage of colistin and antibiotics used in colistin combinations, treatment durations, clinical responses, bacterial eradication and mortality rates were recorded. The study was approved by the Ethics Committee of Gazi University Medical Faculty (date: 12 January 2015; serial no: 25901600-79; decision number: 05).

The study included patients: who were diagnosed with nosocomial pneumonia/VAP caused by MDR-AB as detected in endotracheal aspirates, bronchoalveolar lavage or sputum cultures; who received colistin/colistin combination therapy for at least 72 hours; and who received proper and efficient treatment for other concomitant infection sites and agents. If there was more than one nosocomial pneumonia/ VAP episode caused by MDR-AB during the follow-up of the patient, data of the first infection episode were included in the study.

The study excluded patients: who received colistin therapy for $<72$ hours; in whom colistin use was contraindicated; and who were under the age of 18 .

The Centers for Disease Control and Prevention criteria were used to diagnose nosocomial pneumonia/VAP. ${ }^{[8]}$ The severity of the patient's clinical condition at admission was determined using Acute Physiology and Chronic Health Evaluation (APACHE) II and Sequential Organ Failure Assessment (SOFA) scores, and the severity of underlying diseases was established using the Charlson Comorbidity Index $(\mathrm{CCl}){ }^{[5]}$

\section{Criteria for evaluating clinical response to colistin therapies}

Clinical responses were evaluated at the end of the treatment.

- Complete clinical response (cure): The improvement of all symptoms, signs and laboratory values related to the infection (such asfever, hypothermia, tachypnoea, tachycardia, leukocytosis, leukopenia, C-reactive protein and procalcitonin).

- Partial clinical response: The improvement of only a portion of the symptoms, signs, and laboratory values observed at the beginning of the infection.

- Treatment failure: The non-improvement or worsening of the symptoms, signs and laboratory values related to the infection despite the antimicrobial treatment. ${ }^{[5,6]}$

- Microbiological eradication: Throughout treatment, microbiological sampling was conducted every 4 days. Eradication was considered as bacterial absence in two consecutive samples from respiratory tract secretions during or at the end of the treatment. ${ }^{[9]}$

Respiratory tract specimens taken from the ICU patients were sent to the Infectious Diseases and Clinical Microbiology Laboratory of our hospital. Here, the samples were inoculated into blood agar and eosin methylene-blue lactose sucrose agar, and the antibiotic susceptibilities were studied in Mueller-Hinton agar medium using the Kirby-Bauer Disk Diffusion method in accordance with the standards of Clinical and Laboratory Standards Institute for microorganisms growing after $24-48$ hours. In addition to the classical methods for bacteria identification, BBL Crystal Enteric/ Non-Fermentative ID and BBL Crystal Gram-Positive ID kits (Becton Dickinson, USA) were used. The agents/microorganisms and antibiogram results were recorded.

\section{Renal toxicity}

Renal toxicity was defined according to the RIFLE (risk, injury, failure, loss and end-stage) criteria, and a basal serum creatinine
( $\mathrm{SCr}$ ) value $\leq 114.9 \mu \mathrm{mol} / \mathrm{L}$ was considered normal renal function. Nephrotoxicity was defined as an increase in basal $\mathrm{SCr}$ value $>50 \%$ or the need for renal replacement therapy during the treatment. ${ }^{[5,10]}$

\section{Statistical analysis}

Statistical analysis was conducted using SPSS version 17 (IBM, USA). Continuous data were expressed in median and interquartile range (IQR) values; categorical data were expressed in numbers and percentages. Continuous data of the patients who survived and died at the end of the study were compared using the Mann-Whitney $U$-test, and categorical data were compared using the $\chi^{2}$ test. Logistic regression analysis was conducted with the significant data from the univariate analysis in order to determine independent risk factors for mortality. Additionally, logistic regression analysis was conducted to determine the effect of colistin monotherapy and combination therapies on mortality. Statistical significance level was considered for $p<0.05$.

\section{Results}

The study included 134 patients, median age $68(58.0-76.3)$ years and $53.3 \%$ male. Median admission SOFA score was 7 ( 5 - 11), and median discharge/date-of-death SOFA score was 14 (8-17). Admission to ICU was mostly due to respiratory insufficiency (66.7\%) and sepsis/septic shock (54.8\%). The most common comorbidity was hypertension (52.6\%). A total of 118 (87.4\%) and 122 (90.4\%) patients received mechanical ventilation (MV) support at admission and during the A. baumannii infection, respectively. A total of 112 (83.6\%) patients died during the study period. The discharge/day-of-death SOFA score and procalcitonin value were higher in those who died (15 v. $4, p<0.0001$ and $0.72 \mathrm{ng} / \mathrm{mL}$ v. $3.35 \mathrm{ng} / \mathrm{mL}, p<0.0001$ ) (Table 1).

Mortality was lower in patients who received non-invasive MV (NIMV) support during infection ( $p=0.010$ ), but higher in patients who: received MV support at admission $(p=0.002)$; received invasive $M V$ (IMV) support at admission $(p<0.0001)$; received MV support during infection $(p<0.0001)$; received IMV support during infection $(p<0.0001)$; received vasopressor support; had a central venous catheter and arterial catheter during infection $(p<0.0001)$; developed septic shock $(p<0.0001)$; received sedation ( $p=0.046)$; had higher $\mathrm{CCl}(p=0.049)$; and had haematological malignancy $(p=0.017$ ) (Tables 1 and 2 ).

In our study, $98.5 \%$ of $A$. baumannii strains were susceptible to colistin. Colistin-carbapenem combination therapy was administered to $60 \%$ of the patients, and mortality rates were higher $(p=0.016)$ in this group. Microbiological eradication was achieved in $11 / 42$ patients receiving colistin-sulbactam/ampicillin combination therapy, and mortality was lower in these patients ( $p=0.007$ ) (Table 3 ).

The patients were divided into two groups, namely clinically responsive (complete response/cure or partial response) and treatment failure, according to our definitions. There was no difference in clinical response between patients treated with colistin and colistin combination therapies (Table 4).

Mortality was higher in patients with: higher procalcitonin and lower albumin levels prior to the colistin/combination therapy ( $p=0.007$ and $p=0.001$, respectively); lower mean arterial blood pressure levels $(p<0.0001)$; and higher procalcitonin levels, higher pulse rates and higher creatinine levels after colistin/combination therapy ( $p<0.0001, p=0.009$ and $p=0.004$, respectively). Mortality was lower in patients with higher haemoglobin and albumin levels after colistin/combination therapy $(p<0.001$ and $p<0.0001$, respectively) (Table 5). 
Table 1. General characteristics of the patients

\begin{tabular}{|c|c|c|c|c|}
\hline Characteristics & $\begin{array}{l}\text { Total } \\
(N=134), n(\%)\end{array}$ & $\begin{array}{l}\text { Survived } \\
(N=22), n(\%)\end{array}$ & $\begin{array}{l}\text { Died } \\
(N=112), n(\%)\end{array}$ & $p$-value \\
\hline Age (years), median (IQR) & $68(58.0-76.3)$ & $66(47.5-75.0)$ & $68.5(60.3-77.8)$ & 0.20 \\
\hline Sex (male) & $72(53.7)$ & $9(41.0)$ & $63(56.3)$ & 0.24 \\
\hline APACHE II, median (IQR) & $23(19.0-27.0)$ & $21.5(18.8-26)$ & $24(19-27)$ & 0.21 \\
\hline Admission SOFA, median (IQR) & $7(5-11)$ & $6(3-12)$ & $8(6-11)$ & 0.09 \\
\hline Discharged/day-of-death SOFA, median (IQR) & $14(8-17)$ & $4(2-6)$ & $15(12-17)$ & 0.0001 \\
\hline Admission procalcitonin $(\mathrm{ng} / \mathrm{mL})$, median (IQR) & $1.9(0.5-8.0)$ & $0.8(0.3-5.5)$ & $2.0(0.6-8.1)$ & 0.25 \\
\hline Discharged/day-of-death procalcitonin $(\mathrm{ng} / \mathrm{mL})$, median (IQR) & $2.4(0.7-9.6)$ & $0.7(0.3-1.5)$ & $3.4(1.3-10.1)$ & $<0.0001$ \\
\hline GCS, median (IQR) & $10(7-14)$ & $11(8-15)$ & $10(7-14)$ & 0.36 \\
\hline \multicolumn{5}{|l|}{ Admission site } \\
\hline Emergency service & $56(41.8)$ & $7(31.8)$ & $49(43.8)$ & 0.35 \\
\hline Internal medicine ward & $55(41)$ & $9(40.9)$ & $46(41.1)$ & 1 \\
\hline Other wards & $18(13.4)$ & $4(18.2)$ & $14(12.5)$ & 0.48 \\
\hline Other hospital & $5(3.7)$ & $2(9.1)$ & $3(2.7)$ & 0.15 \\
\hline \multicolumn{5}{|l|}{ Comorbidites } \\
\hline Diabetes mellitus & $40(29.8)$ & $5(22.7)$ & $35(31.3)$ & 0.61 \\
\hline Cardiovascular diseases & 45 (33.6) & $6(27.3)$ & $39(34.8)$ & 0.62 \\
\hline Hypertension & $71(53.0)$ & $9(40.9)$ & $62(55.4)$ & 0.25 \\
\hline Solid malignancy & $23(17.2)$ & $2(9.1)$ & $21(18.8)$ & 0.27 \\
\hline Haematological malignancy & $24(18.0)$ & - & $24(21.4)$ & 0.02 \\
\hline Chronic pulmonary diseases & $29(21.6)$ & $5(22.7)$ & $24(21.4)$ & 0.89 \\
\hline Chronic kidney diseases & $41(30.6)$ & $5(22.7)$ & $36(32.1)$ & 0.46 \\
\hline Liver failure & $7(5.2)$ & - & $7(6.3)$ & 0.23 \\
\hline Neurological diseases & $22(16.4)$ & $3(13.6)$ & $19(17.0)$ & 0.70 \\
\hline $\mathrm{CCl}$, median (IQR) & $3(2.0-4.0)$ & $2(1.0-4.0)$ & $3(2.0-4.8)$ & 0.049 \\
\hline \multicolumn{5}{|l|}{ Main diagnosis at admission } \\
\hline Respiratory insufficiency & $90(67.2)$ & $13(59.1)$ & $77(68.8)$ & 0.46 \\
\hline Sepsis/septic shock & $74(55.2)$ & $10(45.5)$ & $64(57.1)$ & 0.35 \\
\hline Cardiovascular disorders & $14(10.4)$ & $3(13.6)$ & $11(9.8)$ & 0.75 \\
\hline Neurological disorders & $7(5.2)$ & $2(9.1)$ & $5(4.5)$ & 0.37 \\
\hline Metabolical disorders & $5(3.7)$ & $1(4.5)$ & $4(3.6)$ & 0.83 \\
\hline Gastrointestinal/hepatological disorders & $9(6.7)$ & $1(4.5)$ & $8(7.2)$ & 0.31 \\
\hline Renal insufficiency & $30(22.4)$ & $5(22.7)$ & $25(22.3)$ & 0.97 \\
\hline Postarrest & $4(3)$ & $1(4.5)$ & $3(2.7)$ & 0.64 \\
\hline Postoperative respiratory insufficiency & $1(0.7)$ & - & $1(0.9)$ & - \\
\hline Trauma & $1(0.7)$ & - & $1(0.9)$ & - \\
\hline Length of stay in hospital (days), median (IQR) & $29(17.0-43.8)$ & $31(19.0-41.5)$ & $28(16.0-47.5)$ & 0.50 \\
\hline Length of stay in ICU (days), median (IQR) & $15.5(8.0-30.3)$ & $16.5(8.0-27.5)$ & $15(8.0-30.8)$ & 0.74 \\
\hline Mortality at the 28th day & $83(61.9)$ & $0(0)$ & $83(74.1)$ & $<0.0001$ \\
\hline Mortality at the 90th day & $109(81.3)$ & $0(0)$ & $109(97.3)$ & $<0.0001$ \\
\hline GCS = Glasgow Coma Scale; IQR = interquartile range. & & & & \\
\hline
\end{tabular}

Nephrotoxicity developed in 26 (19.3\%) patients on median day 6 of colistin therapy. Mortality was lower in patients with a RIFLE score of 'no risk' $(p=0.009)$ and higher in patients with a RIFLE score of 'failure' $(p=0.004)$ at the end of colistin therapy (Table 5).
A logistic regression analysis was conducted using data significant on univariate analysis: discharge/day-of-death SOFA score, invasively mechanically ventilated during infection, receiving vasopressors, use of colistin-carbapenem combination, microbiological eradication with colistin- sulbactam/ampicillin, RIFLE score of 'failure' at the end of colistin therapy, post-treatment mean blood pressure and post-treatment albumin levels. Discharged/ day-of-death SOFA score and receiving vasopressor support were independent risk factors ( $p=0.001$, odds ratio (OR) $2.02,95 \%$ 
Table 2. Characteristics of patients during intensive care follow-up

\begin{tabular}{|c|c|c|c|c|}
\hline Characteristics & $\begin{array}{l}\text { Total } \\
(N=134), n(\%)\end{array}$ & $\begin{array}{l}\text { Survived } \\
(N=22), n(\%)\end{array}$ & $\begin{array}{l}\text { Died } \\
(N=112), n(\%)\end{array}$ & $p$-value \\
\hline MV on admission & $118(88.1)$ & $15(68.2)$ & $103(92.0)$ & 0.002 \\
\hline IMV on ICU admission & $112(83.6)$ & $12(54.5)$ & $100(89.3)$ & 0.0001 \\
\hline Duration of IMV (days), median (IQR) & $12(6.0-30.6)$ & $8.5(3.3-28.0)$ & $12(7.0-30.8)$ & 0.330 \\
\hline NIMV on ICU admission & $26(19.4)$ & $5(22.7)$ & $21(18.8)$ & 0.660 \\
\hline Duration of NIMV (days), median (IQR) & $2(1.0-7.0)$ & $7(5.0-8.0)$ & $2(1.0-4.3)$ & 0.016 \\
\hline Duration of total MV (days), median (IQR) & $12(7.0-30.0)$ & $9(5.0-19.0)$ & $12.5(7.0-30.3)$ & 0.260 \\
\hline MV at time of infection & $122(91.0)$ & $15(68.2)$ & $107(95.5)$ & 0.0001 \\
\hline Duration of MV (days), median (IQR) & $8(4.0-18.3)$ & $8(4.0-13.0)$ & $9(4.0-19.0)$ & 0.690 \\
\hline IMV during A. baumannii infection & $109(81.3)$ & $11(50.0)$ & $98(87.5)$ & 0.0001 \\
\hline NIMV during A. baumannii infection & $12(8.9)$ & $5(22.7)$ & $7(6.3)$ & 0.010 \\
\hline Tracheostomised & $28(20.9)$ & $3(13.6)$ & $25(22.3)$ & 0.360 \\
\hline \multicolumn{5}{|l|}{ Other characteristics } \\
\hline Vasopressor support & $102(76.1)$ & $3(13.6)$ & $99(88.4)$ & 0.0001 \\
\hline Central venous catheterisation & $117(87.3)$ & $14(63.6)$ & $103(92.0)$ & 0.0001 \\
\hline Arterial catheterisation & $104(77.6)$ & $4(18.2)$ & $100(89.3)$ & 0.0001 \\
\hline Septic shock & $90(67.2)$ & $1(4.5)$ & $89(79.5)$ & 0.0001 \\
\hline Sedation & $44(32.8)$ & $3(13.6)$ & 41 (36.6) & 0.0460 \\
\hline
\end{tabular}

confidence interval (CI) $1.33-3.06$ and $p=0.020$, OR $9.01,95 \% \mathrm{Cl}$ 1.37 - 59.46, respectively) for ICU mortality (Table 6).

Logistic regression analysis was conducted based only on treatments in order to investigate the effects of colistin monotherapy and combination therapies on mortality. Receiving colistincarbapenem therapy was shown to be an independent risk factor for mortality ( $p=0.020$, OR $3.28,95 \% \mathrm{Cl} 1.27-8.48)$.

\section{Discussion}

In ICUs, MDR-AB infection is an important health issue, and is associated with $8-43 \%$ mortality. ${ }^{[1,11,12]}$ Pneumonia is the most common type of infection; its mortality rate can increase up to $65 \%{ }^{[4,13]}$ when attributed to MDR-AB. In our ICU, the mortality rate was $83.6 \%$ in 134 patients with MDR-AB-associated pneumonia/VAP. The higher mortality rates of the present study compared with those in the literature were attributed to comorbid diseases, infections and the advanced age of our patients.

Based on European surveillance data from 2009, A. baumannii infections in ICUs occured in cases of pneumonia at a rate of $21.8 \%$, in bloodstream infections at a rate of $17.1 \%$, and in urinary system infections at a rate of $11.9 \% .^{[3,14,15]}$ Based on Gazi University Medical Faculty Hospital's Infection Control Committee data of 2002, the incidence of $A$. baumannii as an agent for infections in all ICUs was $27.2 \%$, and $77 \%$ of these cases are pneumonia/VAP.

A study by Dizbay et al., ${ }^{[11]}$ which was conducted in our hospital, found that $80.5 \%$ of $A$. baumannii isolates from VAPs in our ICUs were MDR. It is becoming more difficult to treat these infections all over the world due to the emerging antibiotic resistance of $A$. baumannii. In the 1990s, A. baumannii resistance to ceftazidime was $32-45 \%$, and $28-64 \%$ to amikacin, $4-94 \%$ to ciprofloxacin, and $0-2 \%$ to imipenem. ${ }^{[2]}$ However, resistance has increased to $85 \%$ for ceftazidime, $90 \%$ for ciprofloxacin, $38-71 \%$ for imipenem, and $2.7-12 \%$ for tigecycline over the last decade. In contrast, colistin resistance is rarely reported. ${ }^{[2]}$ In the study by Dizbay et al. ${ }^{[1]]}$ the rate of antibiotic resistance in A. baumannii infections was $95.5 \%$ for ciprofloxacin, $72.7 \%$ for cefepime, $80.3 \%$ for imipenem, $17.2 \%$ for meropenem, $68.2 \%$ for cefoperazone/sulbactam, $30.3 \%$ for netilmicin, $25.8 \%$ for tigecycline, and $0 \%$ for colistin. ${ }^{[1]}$ Therefore, colistin is the most frequently used antibiotic for A. baumannii infections in our hospital.

Colistin has a bactericidal effect based on concentration. It has low pulmonary penetration, but intravenous colistin is effective in MDR$A B$ pneumonia infections; however, the adequacy of monotherapy is doubtful. ${ }^{[1]}$ Although MDR-AB strains are generally susceptible to colistin, colistin monotherapy may cause in vitro heteroresistance. Combination therapies are recommended to avoid such resistance. Studies comparing colistin monotherapy and combined therapies provide inconsistent results, with insufficient evidence supporting the superiority of combined therapies. ${ }^{[3,5,16]}$ Colistin is usually combined with sulbactam, cephalosporins, carbapenems, piperacillintazobactam, monobactams, aminoglycosides, fluoroquinolones, rifampin, tetracyclines and tigecycline. ${ }^{[1,4,5,17]}$

Two studies have achieved a positive clinical response of 73.0 $80.8 \%$ and a microbiological response of $94.9 \%$ when using colistin in the treatment of MDR Gram-negative infections. ${ }^{[18,19]}$ Kallel et al. ${ }^{[7]}$ achieved a positive clinical response of $60 \%$ with colistin in the treatment of pneumonia associated with resistant $A$. baumannii and Pseudomonas aeruginosa. The clinical response rate varies between 38 and $57 \%$ and the microbiological response rate varies between 45 and $69 \%$ in pneumonia treatment with colistin therapy ${ }^{[4]}$ One study, which compared colistin doses in patients with A. baumannii infection, found a clinical response of $70.8 \%$ and a microbiological response of $62.5 \% \cdot{ }^{[6]}$ Another study found an efficacy of $45-88 \%$ for colistin. ${ }^{[20]}$ One prospective study conducted in 28 hospitals compared colistin, sulbactam, tigecycline and tetracycline monotherapies and their combinations. Monotherapy was administered to $67.3 \%$ and combination therapy was administered to $32.7 \%$ of 101 patients included in the study. The study concluded no difference between 
Table 3. Antibiotic susceptibility, treatment modalities, clinical response and bacterial eradication

\begin{tabular}{|c|c|c|c|c|}
\hline Characteristics & $\begin{array}{l}\text { Total } \\
(N=134), n(\%)\end{array}$ & $\begin{array}{l}\text { Survived } \\
(N=22), n(\%)\end{array}$ & $\begin{array}{l}\text { Died } \\
(N=112), n(\%)\end{array}$ & $p$-value \\
\hline Day of $A$. baumanii isolation after admission, median (IQR) & $5(2.8-11.0)$ & $4.5(1.0-9.5)$ & $5(3.0-11.0)$ & 0.23 \\
\hline Duration of colistin use (days), median (IQR) & $9(4-17)$ & $11(7-18)$ & $9(4-17)$ & 0.46 \\
\hline \multicolumn{5}{|l|}{ Antibiotic susceptibility of $A$. baumanni } \\
\hline Colistin & $132(98.5)$ & $22(100)$ & $110(98.2)$ & 0.53 \\
\hline Tigecycline & $60(44.8)$ & $8(36.4)$ & $52(46.4)$ & 0.48 \\
\hline Sulbactam/ampicillin & $18(13.4)$ & $2(9.1)$ & $16(14.3)$ & 0.51 \\
\hline Aminoglycosides & $43(32.1)$ & $6(27.3)$ & $37(33)$ & 0.80 \\
\hline Carbapenems & $6(4.5)$ & - & $6(5.4)$ & 0.27 \\
\hline Quinolones & $8(5.9)$ & $2(9.1)$ & $6(5.4)$ & 0.50 \\
\hline Trimethoprim/sulphamethoxazole & $5(3.7)$ & $1(4.5)$ & $4(3.6)$ & 0.83 \\
\hline \multicolumn{5}{|l|}{ Antibiotic treatment } \\
\hline Colistin monotherapy & $23(17.2)$ & $3(13.6)$ & $20(17.9)$ & 0.63 \\
\hline Colistin-carbapenems & $80(59.7)$ & $8(36.4)$ & $72(64.3)$ & 0.02 \\
\hline Colistin-sulbactam/ampicillin & $42(31.3)$ & $7(31.8)$ & $35(31.3)$ & 1 \\
\hline Colistin-tigecycline & $26(19.4)$ & $2(9.1)$ & $24(21.4)$ & 0.18 \\
\hline Colistin-cefoperazone/sulbactam & $17(12.7)$ & $2(9.1)$ & $15(13.4)$ & 0.45 \\
\hline \multicolumn{5}{|l|}{ Duration of antibiotic treatment (days), median (IQR) } \\
\hline Colistin monotherapy & $4(2.0-7.0)$ & $4(2.0-7.0)$ & $4(2.0-9.5)$ & 0.93 \\
\hline Colistin-carbapenems & $7(3.0-11.0)$ & $14(8.5-15.0)$ & $6(3.0-9.5)$ & 0.01 \\
\hline Colistin-sulbactam/ampicillin & $7.5(4.0-12.0)$ & $8(6.0-8.0)$ & $7(4.0-13.0)$ & 0.93 \\
\hline Colistin-tigecycline & $5(2.0-12.0)$ & $8.5(6.0-11.0)$ & $4(2.0-12.0)$ & 0.53 \\
\hline Colistin-cefoperazone/sulbactam & $6(3.0-12.0)$ & $3(2.0-4.0)$ & $6(3.5-13.5)$ & 0.12 \\
\hline \multicolumn{5}{|l|}{ Bacterial eradication rates } \\
\hline Colistin monotherapy $(n=23)$ & $3(2.2)$ & - & $3(2.7)$ & 0.44 \\
\hline Colistin-carbapenems $(n=80)$ & $7(5.2)$ & - & $4(3.6)$ & 0.05 \\
\hline Colistin-sulbactam/ampicillin ( $n=42)$ & $11(8.2)$ & $5(22.7)$ & $6(5.4)$ & 0.007 \\
\hline Colistin-tigecycline $(n=26)$ & $2(1.5)$ & $1(4.5)$ & $1(0.9)$ & 0.20 \\
\hline Colistin-cefoperazone/sulbactam $(n=17)$ & - & - & - & - \\
\hline
\end{tabular}

Table 4. Effectiveness of antibiotics in the treatment of patients

\begin{tabular}{lll}
\hline Antibiotic & $\begin{array}{l}\text { Clinical responsiveness } \\
\text { (complete and partial } \\
\text { response), } \boldsymbol{n}(\%)\end{array}$ & $\begin{array}{l}\text { Treatment failure, } \\
\boldsymbol{n}(\%)\end{array}$ \\
\hline Colistin monotherapy $(n=23)$ & $6(26.1)$ & $17(73.9)$ \\
Colistin-carbapenem $(n=80)$ & $21(26.2)$ & $59(73.8)$ \\
Colistin-sulbactam/ampicillin $(n=42)$ & $14(33.3)$ & $28(66.7)$ \\
Colistin-tigecycline $(n=26)$ & $7(27.0)$ & $19(73.0)$ \\
Colistin-cefoperazone/sulbactam $(n=17)$ & $5(29.4)$ & $12(70.6)$ \\
Colistin-sulbactam $(n=53)$ & $19(35.8)$ & $34(64.2)$
\end{tabular}

monotherapy and combined therapies in sepsis caused by resistant $A$. baumannii. ${ }^{[3]}$ In our study, colistin monotherapy was used at a rate of $17.2 \%$ and combination therapies were generally preferred. Clinical response rate varied between 26.1 and $33.3 \%$ in colistin and its combinations, which is very low. No significant difference was found in clinical response between colistin and its combinations.

Although there are many studies on the combined use of colistin in the literature, very different results have been reported with similar combinations. There are some studies that report no additional benefit provided by combination therapy, while others report superiority for combined therapy. ${ }^{[5]}$ One study did not find any difference in 28-day mortality between the groups that received colistin-sulbactam, colistin-tigecycline, and colistin-carbapenem. ${ }^{[4]}$ Another study found higher treatment efficacy and survival rates in combined therapy group than monotherapy group; however, there was no significant difference when comparing the combination groups among themselves. ${ }^{[5]}$ In contrast, two randomised controlled trials found no difference in mortality and cure rates despite the higher microbiological eradication rate with combined therapy, when comparing colistin and colistin-rifampicin for treating VAP and several severe infections. ${ }^{[3,21,22]}$ Another study reported no clinical or statistical difference between monotherapy and combined therapy for the treatment 
Table 5. Characteristics of patients, renal functions and colistin nephrotoxity

\begin{tabular}{|c|c|c|c|c|}
\hline & $\begin{array}{l}\text { Total } \\
(N=134), n(\%)\end{array}$ & $\begin{array}{l}\text { Survived } \\
(N=22), n(\%)\end{array}$ & $\begin{array}{l}\text { Died } \\
(N=112), n(\%)\end{array}$ & $p$-value \\
\hline Dialysis before colistin treatment & $57(42.5)$ & $6(27.3)$ & $51(45.5)$ & 0.16 \\
\hline Dialysis during colistin treatment & $69(51.5)$ & $7(31.8)$ & $62(55.4)$ & 0.06 \\
\hline Colistin-related nephrotoxicity & $26(19.4)$ & $2(9.1)$ & $24(21.4)$ & 0.18 \\
\hline ICU day that colistin nephrotoxicity developed & $6(3.0-11.5)$ & $10(10.0-10.0)$ & $6(3.0-12.0)$ & 0.47 \\
\hline Daily dose of colistin (mg), median (IQR) & $150(150.0-287.5)$ & $150(150.0-300.0)$ & $150(150.0-275.0)$ & 0.69 \\
\hline Total dose of colistin (mg), median (IQR) & $1350(450.0-2681.0)$ & $1275(0-2587.5)$ & $1350(450.0-2793.7)$ & 0.46 \\
\hline \multicolumn{5}{|l|}{ RIFLE on ICU admission } \\
\hline No risk & $33(24.6)$ & $7(31.8)$ & $26(23.2)$ & 0.41 \\
\hline Risk & $34(25.4)$ & $7(31.8)$ & $27(24.1)$ & 0.43 \\
\hline Injury & $17(12.7)$ & $1(4.5)$ & $16(14.3)$ & 0.21 \\
\hline Failure & $24(17.9)$ & $2(9.1)$ & $22(19.6)$ & 0.24 \\
\hline Lost & $2(1.5)$ & - & $2(1.8)$ & 0.53 \\
\hline End stage & $24(17.9)$ & $5(22.7)$ & $19(17.0)$ & 0.52 \\
\hline \multicolumn{5}{|l|}{ RIFLE at the begining of colistin treatment } \\
\hline No risk & $30(22.4)$ & $8(36.4)$ & $22(19.6)$ & 0.09 \\
\hline Risk & $23(17.2)$ & $5(22.7)$ & $18(16.1)$ & 0.45 \\
\hline Injury & $14(10.4)$ & $2(9.1)$ & $12(10.7)$ & 0.82 \\
\hline Failure & $34(25.4)$ & $2(9.1)$ & $32(28.6)$ & 0.06 \\
\hline Lost & $6(4.8)$ & - & $6(5.4)$ & 0.267 \\
\hline End stage & $27(20.1)$ & $5(22.7)$ & $22(19.6)$ & 0.74 \\
\hline \multicolumn{5}{|l|}{ RIFLE at the end of colistin treatment } \\
\hline No risk & $19(14.2)$ & $7(31.8)$ & $12(10.7)$ & 0.009 \\
\hline Risk & $21(15.7)$ & $6(27.3)$ & $15(13.4)$ & 0.10 \\
\hline Injury & $16(11.9)$ & $2(9.1)$ & $14(12.5)$ & 0.65 \\
\hline Failure & $40(29.9)$ & $1(4.5)$ & $39(34.8)$ & 0.004 \\
\hline Lost & $9(6.7)$ & $1(4.5)$ & $8(7.1)$ & 0.66 \\
\hline End stage & $29(21.6)$ & $5(22.7)$ & $24(21.4)$ & 0.89 \\
\hline \multicolumn{5}{|l|}{ Laboratory and vital signs } \\
\hline \multicolumn{5}{|l|}{ Before colistin treatment, median (IQR) } \\
\hline Leukocytes $(/ \mu \mathrm{L})$ & $9900(6630-14605)$ & $10975(8240-16887)$ & $9880(5980-14410)$ & 0.21 \\
\hline Haemoglobin (g/L) & $88.0(77.0-104.0)$ & $98.5(85.5-106.0)$ & $86.0(76.0-104.0)$ & 0.05 \\
\hline C-reactive protein (mg/L) & $1315(415-1945)$ & $1660(845-2810)$ & $1230(250-1930)$ & 0.23 \\
\hline Creatinine $(\mu \mathrm{mol} / \mathrm{L})$ & $145.8(70.7-265.2)$ & $92.8(57.4-322.6)$ & $167.9(70.7-265.2)$ & 0.27 \\
\hline Albumin $(g / L)$ & $24.0(20.4-28.0)$ & $26.5(24.8-32.0)$ & $23.0(20.0-27.0)$ & 0.001 \\
\hline Procalcitonin (ng/mL) & $1.4(0.5-5.2)$ & $0.5(0.1-2)$ & $1.6(0.6-6.3)$ & 0.007 \\
\hline Respiratory rate (/min) & $26.0(22.0-30.0)$ & $25.5(22.0-29.3)$ & $26.0(22.0-30.0)$ & 0.84 \\
\hline Temperature $\left({ }^{\circ} \mathrm{C}\right)$ & $36.7(36.5-37.5)$ & $36.8(36.7-37.6)$ & $36.7(36.4-37.5)$ & 0.53 \\
\hline Mean arterial pressure $(\mathrm{mmHg})$ & $65.0(57.5-73.0)$ & $73.5(67.75-86.5)$ & $63.0(55.0-72.0)$ & 0.0001 \\
\hline Heart rate (/min) & $110.0(98.0-125.0)$ & $107.5(94.3-112.5)$ & $110.0(98.0-172.0)$ & 0.12 \\
\hline \multicolumn{5}{|l|}{ After colistin treatment, median (IQR) } \\
\hline Leukocytes $(/ \mu \mathrm{L})$ & $10420(7195-15355)$ & $9820(7570-11320)$ & $10765(6825-15945)$ & 0.55 \\
\hline Haemoglobin (g/L) & $82.5(72.0-95.0)$ & $95.0(84.7-108.5)$ & $80.0(71.0-93.2)$ & 0.001 \\
\hline C-reactive protein (mg/L) & $870(230-1350)$ & $440(201-1375)$ & $910(230-1350)$ & 0.51 \\
\hline Creatinine $(\mu \mathrm{mol} / \mathrm{L})$ & $185.6(97.2-307.6)$ & $97.2(53.0-179.0)$ & $203.3(101.6-313.8)$ & 0.004 \\
\hline Albumin (g/L) & $21(18-25)$ & $27(25-30)$ & $20(17-23)$ & 0.0001 \\
\hline Procalcitonin (ng/ml) & $2.4(0.5-9.6)$ & $0.4(0.1-0.7)$ & $4.1(1.3-11.0)$ & 0.0001 \\
\hline
\end{tabular}


Table 5 (continued). Characteristics of patients, renal functions and colistin nephrotoxity

\begin{tabular}{|c|c|c|c|c|}
\hline & $\begin{array}{l}\text { Total } \\
(N=134), n(\%)\end{array}$ & $\begin{array}{l}\text { Survived } \\
(N=22), n(\%)\end{array}$ & $\begin{array}{l}\text { Died } \\
(N=112), n(\%)\end{array}$ & $p$-value \\
\hline Respiratory rate (/min) & $24.0(20.0-28.0)$ & $22.0(20.0-25.3)$ & $24.0(20.0-28.0)$ & 0.13 \\
\hline Temperature $\left({ }^{\circ} \mathrm{C}\right)$ & $36.6(36.2-37.2)$ & $36.6(36.4-36.7)$ & $36.6(36.2-37.3)$ & 0.52 \\
\hline Mean arterial pressure $(\mathrm{mmHg})$ & $52(40.0-71.5)$ & $78(72.8-87.3)$ & $45(39.0-62.0)$ & 0.0001 \\
\hline Heart rate $(/ \mathrm{min})$ & $109.0(88.0-129.0)$ & $94.5(85.75-106.0)$ & $112.0(89.0-132.0)$ & 0.009 \\
\hline
\end{tabular}

Table 6. Independent risk factors affecting ICU mortality

\begin{tabular}{lll}
\hline Parameter & $p$-value & OR $(\mathbf{9 5} \% \mathbf{C I})$ \\
\hline Discharge/day-of-death SOFA score & 0.001 & $2.02(1.33-3.06)$ \\
Vasopressor support & 0.022 & $9.01(1.37-59.46)$
\end{tabular}

of MDR-AB infections, and indicated that use of monotherapy would be reasonable for at least treating less severe infections. ${ }^{[3]}$ The same study suggested that drug choice can be customised according to drug access, drug interactions, side-effects and costs, since there is no difference between the drugs in resistant A. baumannii pneumonia reported to be hospital acquired or ventilator associated. ${ }^{[4]}$

For A. baumannii, the colistin-carbapenem combination is a frequently used combination owing to its high synergistic effect, low antagonism and low resistance. ${ }^{[23]}$ This was also the most commonly used combination in the present study (80 patients). However, carbapenem-resistant $A$. baumannii strains and other Gram-negativeresistant infections increase with the use of carbapenem. ${ }^{[5,14]}$ Previous studies from Turkey have reported 55 - 70\% carbapenem resistance for A. baumannii..$^{11,24,25]}$ In the present study, carbapenem resistance was $95.5 \%$. In A. baumannii infections resistant to carbapenem, mortality is higher than those with susceptibility to carbapenem. ${ }^{[12]}$ The present study also found higher mortality in patients who received colistin-carbapenem combination. This increased mortality may have resulted either from the failure to achieve the desired synergistic effect after administration to severely infected patients or from the addition of infections by other resistant microorganisms developing under carbapenem.

Sulbactam is an antibiotic with bactericidal or bacteriostatic effects that is used in MDR-AB infections. It is not recommended to use alone for the treatment of severe infections. In vitro studies with imipenem-resistant $A$. baumannii strains report a high synergistic effect of sulbactam with colistin. ${ }^{[1,5,17]}$ Since sulbactam alone has not been available in Turkey, combined preparations of sulbactam/ ampicillin or cefoperazone/sulbactam in high doses have been used. In our hospital, a formula with sulbactam alone has been used for the previous year. The present study also found higher microbiological eradication with a colistin-sulbactam-ampicillin (SAM) combination. However, it did not have an effect on clinical response. A previous study compared colistin and colistin-sulbactam in VAP infections caused by MDR-AB in ICU, and found both similar clinical response and microbiological response on day 5 of treatment and at the end of the treatment. In conclusion, no difference was reported between combination therapy and monotherapy. ${ }^{[26]}$

The known risk factors for $A$. baumannii infections include advanced age, severe underlying diseases, immunosuppression, major traumas, burns, invasive procedures, catheters, MV support, surgical treatments, prolonged hospital stay, and inadequate and improper antibiotic therapies. ${ }^{[1,4,27]}$ Additionally, factors such as underlying diseases, varied infection severity depending on the patient, diagnostic delays and initiation of treatment may also cause difficulties in evaluating the efficacy of combined therapies in resistant $A$. baumannii infections. ${ }^{[5]}$ The present study evaluated APACHE II, CCI, SOFA and RIFLE scores, and found a higher discharged/day-of-death SOFA score associated with increased mortality. Furthermore, a higher $\mathrm{CCl}$ score and the presence of underlying haematological malignancy were established as important factors affecting mortality. One study, which employed colistin combinations in Gram-negative infections, found no difference between treatment regimens, but found mortality was significantly associated with age, $\mathrm{CCl}$ score and severity of acute disease. ${ }^{[28]}$ Similar to the present study, other researchers have found higher mortality in patients with malignancy and chronic renal damage. ${ }^{[4]}$

Colistin-related nephrotoxicity rates varying between 0 and $33 \%$ have been reported in previous studies. Colistin nephrotoxicity usually occurs within the first week of use. Age, severity of the underlying disease, co-existence of septic shock, use of other nephrotoxic agents, time of exposure to colistin and cumulative dose are reported to be associated with colistin nephrotoxicity. ${ }^{[6,20,29]}$ In the present study, colistin-related nephrotoxicity occurred at a rate of $19.2 \%$ and on median day 6 . No correlation was found between exposure time or cumulative time and nephrotoxicity. A direct correlation was not demonstrated between nephrotoxicity and mortality; however, mortality was lower in the 'no risk' group and higher in the 'failure' group based on RIFLE scoring at the end of colistin therapy.

\section{Study limitations}

The present study had some limitations. It was a small-sample, single-centre, retrospective study, which limits generalisability. We did not include trauma patients and surgical patients, since the study was conducted in a medical ICU. The co-existence of other infections with $A$. baumannii infection in the patients posed a challenge, particularly for interpreting the clinical outcome and mortality. It became difficult to interpret the results because of intergroup transitions between colistin monotherapy and combination therapies, or other treatment changes.

\section{Conclusion}

In conclusion, the present study has shown that colistin monotherapy and combination therapies have no superiority over each other for clinical response in the treatment of nosocomial pneumonia/VAP caused by MDR-AB; colistin-SAM was associated with improved microbiological eradication and colistin-carbapenem combination was associated with increased mortality.

\section{References}

1. Karageorgopoulos $D E$, Falagas ME. Current control and treatment of multidrug-resistant Acinetobacter baumannii infections. Lancet Infect Dis 2008;8(12):751-762. [http://dx.doi. org/10.1016/S1473-3099(08)70279-2] 
2. Vila J, Pachón J. Therapeutic options for Acinetobacter baumannii infections. Expert Opin Pharmacother 2008;9(4):587-599. [http://dx.doi.org/10.1517/14656566.9.4.587]

3. López-Cortés LE, Cisneros JM, Fernández-Cuenca F, et al. Monotherapy versus combination therapy for sepsis due to multidrug-resistant Acinetobacter baumannii: Analysis of a multicentre prospective cohort. J Antimicrob Chemother 2014;69(11):3119-3126. [http://dx.doi.org/10.1093/jac/dku233]

4. Khawcharoenporn T, Pruetpongpun N, Tiamsak P, Rutchanawech S, Mundy LM, Apisarnthanarak A. Colistin-based treatment for extensively drug-resistant Acinetobacter baumannii pneumonia. Int J Antimicrob Agents 2014;43(4):378-382. [http://dx.doi.org/10.1016/j.jjantimicag.2014.01.016]

5. Batirel A, Balkan II, Karabay O, et al. Comparison of colistin-carbapenem, colistin-sulbactam and colistin plus other antibacterial agents for the treatment of extremely drug-resistant 1322. [http://dx.doi.org/10.1007/s10096-014-2070-6]

6. Yılmaz GR, Baştuğ AT, But A, et al. Clinical and microbiological efficacy and toxicity of colistin in patients infected with multidrug-resistant gram-negative pathogens. J Infect Chemother 2013;19:57-62. [http://dx.doi.org/10.1007/s10156-012-0451-2]

7. Kallel $H$, Bahloul $M$, Hergafi $L$, et al. Colistin as a salvage therapy for nosocomial infection caused by multidrug-resistant bacteria in the ICU. Int J Antimicrob Agents 2006;28(4):366-369. [http://dx.doi.org/10.1016/j.jiantimicag.2006.07.008]

8. Centers for Disease Control and Prevention. Ventilator-associated Pneumonia (VAP) Event 2014. http://www.cdc.gov/nhsn/PDFs/pscManual/10-VAE_FINAL.pdf (accessed 4 January 2015).

9. Kuo SC, Lee YT, Yang SP, et al. Eradication of multidrug-resistant Acinetobacter baumannii from the respiratory tract with inhaled colistin methanesulfonate: A matched case-control study. Clin Microbiol Infect 2012;18(9):870-876. [http://dx.doi.org/10.1111/j.1469-0691.2011.03682.x]

10. Bellomo R, Ronco C, Kellum JA, Mehta RL, Palevsky P, Acute Dialysis Quality Initiative workgroup. Acute renal failure-definition, outcome measures, animal models, fluid therapy and information technology needs: The Second International Consensus Conference of the Acute Dialysis Quality technology needs: The Second International Consensus Conference of the Acute Dialys]
Initiative (ADQI) Group. Crit Care 2004;8(4):204-212. [http://dx.doi.org/10.1186/cc2872]

11. Dizbay M, Altuncekic A, Sezer BE, Ozdemir K, Arman D. Colistin and tigecycline susceptibility among multidrug-resistant Acinetobacter baumannii isolated from ventilator-associated pneumonia. Int Antimicrob Agents 2008;32(1):29-32. [http://dx.doi.org/10.1016/j.jiantimicag.2008.02.016]

12. Lemos EV, de la Hoz FP, Einarson TR, et al. Carbapenem resistance and mortality in patients with Acinetobacter baumannii infection: Systematic review and meta-analysis. Clin Microbiol Infect 2014;20(5):416-423. [http://dx.doi.org/10.1111/1469-0691.12363]

13. European Centre for Disease Prevention and Control (ECDC). Annual Epidemiological Report Reporting on 2009 Surveillance Data and 2010 Epidemic Intelligence Data. Stockholm, Sweden: ECDC, 2011

14. Falagas $M E$, Kopterides $P$. Review risk factors for the isolation of multi-drug-resistant Acinetobacter baumannii and Pseudomonas aeruginosa: A systematic review of the literature. Hosp Infect 2006;64(1):7-15. [http://dx.doi.org/10.1016/j.jhin.2006.04.015]

15. Gaynes R, Edwards JR, National Nosocomial Infections Surveillance System. Overview of nosocomial infections caused by Gram-negative bacilli. Clin Infect Dis 2005:41(6):848-854 [http://dx.doi.org/10.1086/432803]
16. Falagas ME, Rafailidis $\mathrm{Pl}$, loannidou $\mathrm{E}$, et al. Colistin therapy for microbiologically documented multidrug-resistant Gram-negative bacterial infections: A retrospective cohort study of 258 patients. Int J Microbial Agents 2010;35(2):194-199. [http://dx.doi.org/10.1016/j.jiantimicag.2009.10.005]

17. Michalopoulos A, Falagas ME. Review treatment of Acinetobacter infections. Expert Opin Pharmacother 2010;11(5):779-788. [http://dx.doi.org/10.1517/14656561003596350]

18. Koomanachai $\mathrm{P}$, Tiengrim S, Kiratisin $\mathrm{P}$, Thamlikitkul V. Efficacy and safety of colistin (colistimethate sodium) for therapy of infections caused by multidrug-resistant Pseudomonas aeruginosa and Acinetobacter baumannii in Siriraj Hospital, Bangkok, Thailand. Int J Antimicrob Agents 2007;11(5):402-406. [http://dx.doi.org/10.1016/j.ijid.2006.09.011]

19. Markou N, Apostolakos H, Koumoudiou C, et al. Intravenous colistin in the treatment of sepsis from multiresistant Gram-negative bacilli in critically ill patients. Critical Care 2003;7(5):78-83. [http://dx.doi.org/10.1186/cc2358]

20. Cheng CY, Sheng WH, Wang JY, Chen YC, Chang SC. Safety and efficacy of intravenous colistin (colistin methanesulphonate) for severe multidrug-resistant Gram-negative bacterial infection Int J Antimicrob Agents 2010;35(3):297-300. [http://dx.doi.org/10.1016/j.jiantimicag.2009.11.016]

21. Aydemir $\mathrm{H}$, Akduman D, Piskin N, et al. Colistin vs. the combination of colistin and rifampicin for the treatment of carbapenem-resistant Acinetobacter baumannii ventilator-associated pneumonia. Epidemiol Infect 2013;141(6):1214-1222. [http://dx.doi.org/10.1017/S095026881200194X]

22. Durante-Mangoni E, Signoriello G, Andini R, et al. Colistin and rifampicin compared with colistin alone for the treatment of serious infections due to extensively drug-resistant Acinetobacter baumannii: A multicenter, randomized clinical trial. Clin Infect Dis 2013;57(3):349-358. [http:// dx.doi.org/10.1093/cid/cit253]

23. Zusman O, Avni T, Leibovici L, et al. Systematic review and meta-analysis of in vitro synergy of polymyxins and carbapenems. Antimicrob Agents Chemother 2013;57(10):5104-5111. [http:// dx.doi.org/10.1128/AAC.01230-13]

24. Gür D, Hascelik G, Aydın N, et al. Antimicrobial resistance in Gram-negative hospital isolates: Results of the Turkish HITIT-2 surveillance study of 2007. J Chemother 2009:21(4):383-389. [http://dx.doi.org/10.1179/joc.2009.21.4.383]

25. Kurtoğlu MG, Opuş A, Kaya M, Keşli R, Güzelant A, Yüksekkaya Ş. Bir eğitim ve araştırma hastanesinde klinik örneklerden izole edilen Acinetobacter baumannii suşlarında antibakteriye direnç. Ankem Derg 2011;25:35-41.

26. Kalin G, Alp E, Akin A, Coskun R, Doganay M. Comparison of colistin and colistin/sulbactam for the treatment of multidrug resistant Acinetobacter baumannii ventilator-associated pneumonia. Infection 2014;42(1):37-42. [http://dx.doi.org/10.1007/s15010-013-0495-y]

27. Maragakis LL, Perl TM. Acinetobacter baumannii: Epidemiology, antimicrobial resistance, and treatment options. Clin Infect Dis 2008;46(8):1254-1263. [http://dx.doi.org/10.1086/529198]

28. Crusio R, Rao S, Changawala N, et al. Epidemiology and outcome of infections with carbapenem resistant Gram-negative bacteria treated with polymyxin B-based combination therapy. Scand $J$ Infect Dis 2014;46(1):1-8. [http://dx.doi.org/10.3109/00365548.2013.844350]

29. DeRyke CA, Crawford AJ, Uddin N, Wallace MR. Colistin dosing and nephrotoxicity in a large community teaching hospital. Antimicrob Agents Chemother 2010;54(10):4503-4505. [http:// dx.doi.org/10.1128/AAC.01707-09] 